\title{
SPEGAZZINIAN MELIOLA TYPES
}

\section{F. L. STEVENS}

\section{(WITH PLATES XXIV-XXVI)}

Through the kindness of Dr. CARlo SPEgazzINI, I have received recently a number of original packages containing type specimens of Meliola described earlier by Dr. Spegazzini. In each case the packet bore on the outside copious penciled notes concerning the specimen and careful, delicate drawings of the more significant structures. This collection of types, together with the notes and drawings in particular, are a fine commentary on the work of Dr. SPEgazzINI. When we remember the large volume of his descriptive work and reflect that not only his types but other specimens as well are thus thoroughly and carefully annotated and figured, we are in a position more adequately to recognize the great indebtedness of mycology to him.

The drawings of the present collection have not been published, and in view of the comparative inaccessibility of most of these types, it is desirable that they should be made generally accessible to students by publication.

Permission having been received from Dr. Spegazzini, Dr. Alva Peterson has faithfully copied for me, for publication, the most important of the drawings. Such copying was necessary, owing to the color of the paper upon which the originals were drawn, and the faintness of the penciling, which prohibited direct photographic reproduction. These drawings have been compared by me with the type material and are published herewith with such comments as seem necessary. A permanent celloidin mount ${ }^{r}$ has been made from each specimen and, together with a fragment of the type specimen, is deposited in the herbarium of the University of Illinois. The original specimens have been returned to Dr. Spegazzini at La Plata, Argentine.

Meliola armata Speg. (fig. I).-F. Puigg., Pug. I, no. 23I; Sacc. 9:4I5.

${ }^{2}$ Stevens, F. L., Phytopathology 6:367. rgi6. 
On coriaceous leaves, Myrsine (?), Apiahy, May I888; no. 2382 (type).

The type specimen is heavily overgrown with several parasites. There is considerable variation in the character of the mycelium, which is sometimes straight, sometimes quite crooked. I have not been able to see the mycelial setae around the bases of the perithecia from which this species takes it name.

Meliola argentina Speg. (fig. 2).-Fung. Arg., Pug. I, no. I77; Sacc. I:6r.

On Cyperaceae, Buenos Aires, February r880 (type).

The mycelium is very characteristic, close, dense, somewhat like $M$. manca, but distinguished by its very thick mycelial setae, which are striking, being darker and thicker than the mycelium. They are about ${ }_{5} 5 \mu$ thick at base and over $800 \mu$ long. The capitate hyphopodia are angular, that is, not smooth or echinulate as shown in fig. 2. The type is heavily overgrown by a Coniothyrium.

Meliola brasiliensis Speg. (fig. 3).-Fung. Arg., Pug. IV, no. Ir6; Sacc. I:66.

On leaves of Bignoniaceae (?), Apiahy; no. 1551 (type).

The young perithecia are surrounded by an areola of radiating hyphae. The perithecia also possess short hairs as figured and described by Dr. SpEgazZINI.

Meliola Calva Speg. (fig. 4).-F. Puigg., Pug. I, no. 233; Sacc. 9:4I4.

On Laurinaceae, Apiahy, August I88I (type).

Heavily overgrown with "Podosporium penicillium Speg."

Meliola clavatispora Speg. (fig. 5).-F. Puigg., Pug. I, no. 24I; Sacc. 9:422.

On leaves of Apocynaceae, Apiahy, April r88ı; no. r70r (type).

Perhaps the most striking character is in the sessile, nearly globular capitate hyphopodia.

Meliola coronata Speg. (fig. 6).-F. Guar., Pug. I, no. I75; Sacc. 9:428.

On Luchea divaricata, Guarapi, July I883; no. 3847 (type).

The figure shows perithecial hairs to be more conspicuous than they usually are. 
Meliola Crustacea Speg. (fig. 7).-F. Puigg., Pug. I, no. 235; Sacc. 9:4I3.

On Drymis, Apiahy, r88I (type).

The mycelium forms a compact crustose colony, with the parts decidedly more crowded even than is shown in fig. 7 .

Meliola decidua Speg. (fig. 8).-F. Puigg., Pug. I, no. 240; Sacc. 9:426.

On Convolvulaceae (?), Apiahy, April r888; no. 2344 (type).

The capitate hyphopodia are very irregularly angular.

Meliola delicatula Speg. (fig. 9).-F. Guar., Pug. II, no. 63; Sacc. 9:4I 5 .

On Myrisinus, Sierra de Peribebuy, September I5, I883; no. 3985 (type).

Meliola ERIOphora Speg. (fig. Io).-F. Guar., Pug. II, no. 62; Sacc. 9:4I3.

On Ficus ibapoy, Paraguay, January I 883 (type).

Meliola glabriuscula Speg. (fig. ii).-F. Aliq. Paul., no. 35; Sacc. $22: 48$.

On Photiniae (?), Agua branca, Sao Paulo (type).

Meliola gleditschiae Speg. (fig. I2).-Myc. Argent. VI, no. 1337 .

On Gleditschia amorphoidis, Puerto Leon, Missiones, July 1909 (type).

Meliola guareae Speg. (fig. I3).-Myc. Argent. VI, no. I338.

On Guarea balansa, Puerto Leon, Missiones, August 1909 (type).

Meliola harioti Speg. (fig. I4).-F. Guar. nonn. III, no. 78; Sacc. Ir :267; Gaill. Bull. Soc. Myc. Fr. 8:186. 1892.

On Bignoniaceae, Paraguay, no. I29I (type).

Meliola levipoda Speg. (fig. I5).-F. Guar. nonn., no. 77 (p. 26); Sacc. II:264; Bull. Soc. Myc. Fr. 8:181. I892.

On Aspidosperma quebracho, Yaguaron, Paraguay, November I882; no. $35^{89}$ (type).

Meliola LUdibunda Speg. (fig. I6),-F. Guar. I, no. I78; Sacc. 9:43I.

On Pilocarpus pinnatus, Paraguay, January I882; no. 3489 (type). 
Meliola megalospora Speg. (fig. i 7).-F. Arg., Pug. IV, no. II 5 ; Sacc. $1: 67$.

On Jodina rhombifolia, January i 888 (type).

The very coarse hyphae are quite characteristic.

Meliola melastomacearum Speg. (fig. i8).-F. Puigg., Pug. I, no. 232 ; Sacc. 9:4I4.

On Melastomaceae, no. 2485, Apiahy, May I888 (type).

The mycelium is often less straight than might be assumed from the figure. The oval hyphopodia are characteristic.

Meliola obesa Speg. (fig. ig).-F. Guar., Pug. I, no. I79; Sacc. 9:42I.

On Rutaceae, Piragu Bras, July I883; no. 3834 (type).

Meliola obesula Speg. (fig. 20).-F. Guar. nonn., no. 75; Sacc. II:262.

On Rutaceae, Caa-guaza, Brazil, January I882; no. 3585 (type).

Meliola Puiggari Speg. (fig. 2i).-F. Puigg., Pug. I, no. 228; Sacc. 9:4I4.

On Rubus, Apiahy, May I888; no. 2722 (type).

Meliola pulchella Speg. (fig. 22).-F. Puigg., Pug. I, no. 227 ; Sacc. 9:4I4.

On Myrtaceae, Apiahy, ı88ı; no. r699 (type).

Meliola sapindacearum (fig. 23).-F. Guar. nonn., III, no. 79; Sacc. I I:266; Bull. Soc. Myc. Fr. 8:184. I892.

On Sapindaceae, Caa-guazu, Brazil, January ı882; no. 3600.

Meliola Spegazziniana Wint. (fig. 24).-F. Guar., Pug. II, no. 64; Sacc. 9:4I8.

On Compositae, Paraguari, March 5, I883; no. 375 (type).

Meliola sororcula (fig. 25).-F. Puigg., Pug. I, no. 230; Sacc. 9:4I8.

On Baccharis pingrea, Apiahy, May ı886; no. 2774 (type).

Meliola subcrustacea Speg. (fig. 26).-F. Puigg., Pug. I, no. 236 ; Sacc. 9:430.

Apiahy, I888; no. 2703 (type).

Meliola tabernaemontanae Speg. (fig. 27).-Myc. Argent. VI, no. I345; Bompland Missiones. 


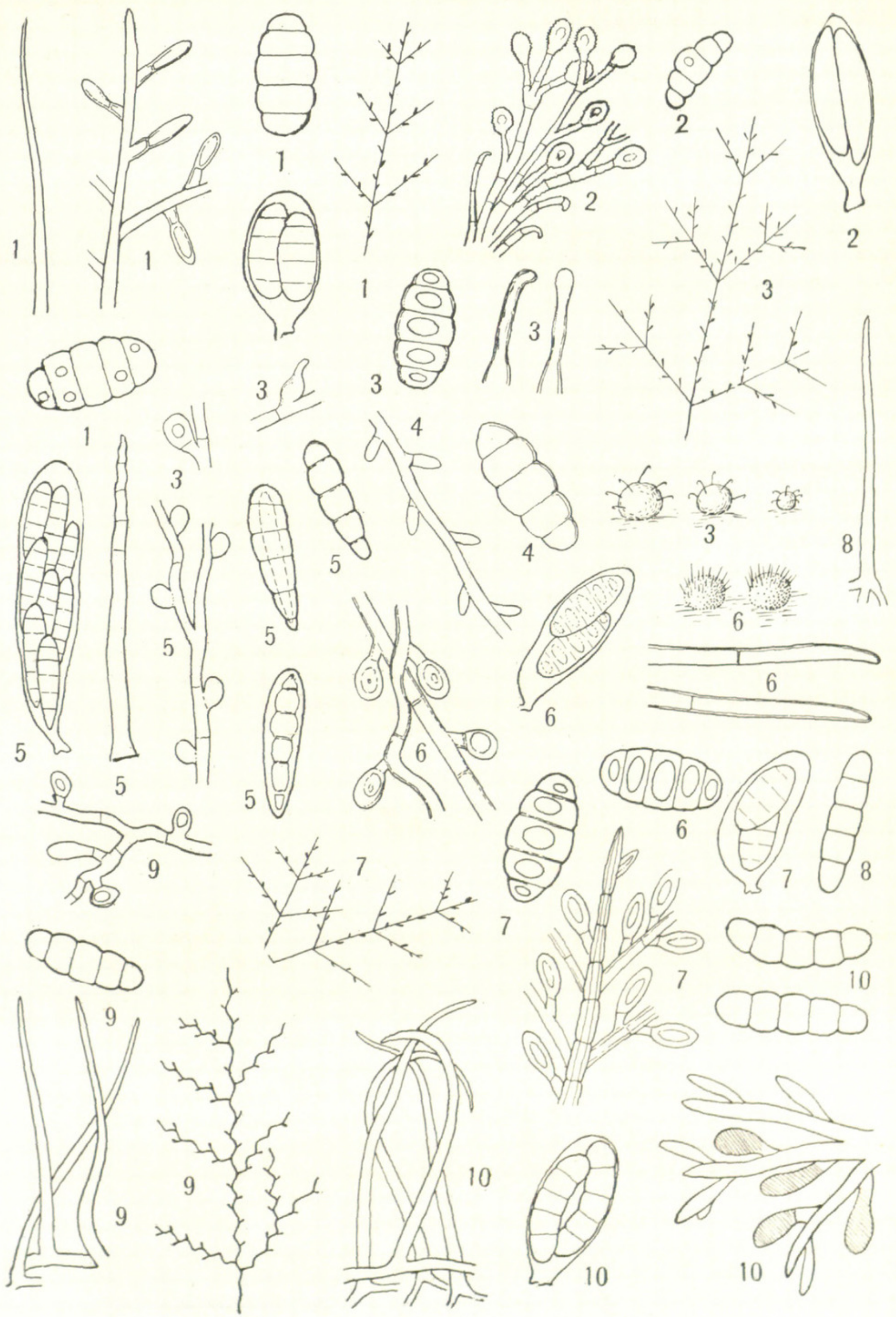

STEVENS on MELIOLA 


\section{$2 \mathrm{BHL}$ Biodiversity Heritage Library}

Stevens, Frank Lincoln. 1917. "Spegazzinian Meliola Types." Botanical gazette 64(5), 421-425. https://doi.org/10.1086/332169.

View This Item Online: https://www.biodiversitylibrary.org/item/109348

DOI: https://doi.org/10.1086/332169

Permalink: https://www.biodiversitylibrary.org/partpdf/223947

\section{Holding Institution}

Missouri Botanical Garden, Peter H. Raven Library

\section{Sponsored by}

Missouri Botanical Garden

\section{Copyright \& Reuse}

Copyright Status: Public domain. The BHL considers that this work is no longer under copyright protection.

This document was created from content at the Biodiversity Heritage Library, the world's largest open access digital library for biodiversity literature and archives. Visit BHL at https://www.biodiversitylibrary.org. 